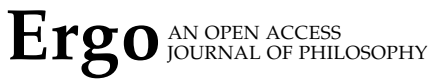

\section{A Semantic Problem For StAge Theory}

\author{
MATTHEW MCKEEVER
}

\begin{abstract}
I subject the semantic claims of stage theory to scrutiny and show that it's unclear how to make them come out true for a simple and deep reason: the stage theorist needs tensed elements to semantically modify the denotations of referring expressions to enable us to talk about past and future stages. But in the syntax of natural language, expressions carrying tense modify verbs and adjectives and not referring expressions. This mismatch between what the stage theorist needs, and what language provides, makes it hard to see how the stage theorist's semantic claims could be true.
\end{abstract}

\section{Introduction}

Stage theory is the metaphysical view that everyday objects like you and the Eiffel tower are stages-instantaneous temporal parts. Existing defenses of stage theory by Katherine Hawley (2001) and Ted Sider (1996: 2001) quite explicitly include certain semantic claims. Thus Sider says that stages are

the referents of ordinary terms, members of ordinary domains of quantification, subjects of ordinary predications, and so on. (2001: 60)

In the same vein, Hawley says,

The two accounts [perdurantism and stage theory] differ over what we talk about when we use phrases like 'the tennis ball', and about which objects satisfy sortal predicates like 'is a tennis ball' ... according to stage theory, it is stages themselves which are tennis balls (or bananas, or human beings, as the case may be). 2001.

Not only that, both agree, in broad outline, about the stage-theoretic truth conditions of sentences. A sentence like

Contact: Matthew McKeever <mipmckeever2@gmail.com> 
(1) John was happy

will be true for the stage theorist provided there's some stage which is suitably related to the stage picked out, at the time of utterance, by 'John', such that it is prior to the time of utterance, and it possesses the property of being happy (Sider 2001, 193; Hawley 2001. 54-57). Sider and Hawley disagree slightly about the nature of suitable relatedness; it is unimportant for this paper, but I will on occasion use 'I-relation' to denote the relation.

My aim here is to examine the cogency of these claims, in light of what our best theories of the compositional semantics of natural language, and in particular tense, tell us. Having briefly reviewed the theory of tense, I will present a very simple sentence which I claim the stage theorist will not be able to deal with and show that this failure arises from a deep mismatch between what the stage theorists need of a semantics of tense and what natural language supplies. The conclusion will be that there's good reason to doubt that the stage theorist will be able to make good on their semantic claims, and thus, to the extent that these claims are an important part of stage theory, good reason to think stage theory false.

\section{The Semantics of Tense}

My aim in this paper is to consider to what extent the stage theorist's semantic claims fit with the main views about the functioning of tense in natural language found in the literature. In this section, I will provide a brief overview of these views.

There are two main views about the semantics of tense. On the one hand, there is the view which has its roots in Priorean tense logic (see, e.g., Prior 1968, or Goranko \& Galton 2015 for an overview) according to which expressions which carry tense marking introduce temporal operators. As a first pass, this would be to say that a sentence like

(2) Carrie swam

is well represented in tense logic as

\section{(3) P (Carrie swim)}

where ' $\mathrm{P}$ ' is a past tense modal operator, the meaning of which could be glossed as 'it has at some time been the case that'. This functions like a possibility operator in alethic modal logic: it operates on an intension (a function from points of evaluation to truth values; in the alethic case these points are worlds and in the temporal case times) and maps it to true provided there is a time prior to 
the utterance time such that the intension maps it to true, which is to say provided there is some time in the past at which the sentence is true. It thus has the force, in the metalanguage in which the tense language is interpreted, of existential quantification over past times (other operators are associated with existential quantification over future times, and yet others with universal quantification over past and future times).

We could then say that there are temporal operators in natural language, so that the syntactic logical form of our sentence would be (simplifying massively)

\section{(4) $[s[\mathrm{P}[$ Carrie swim $]]$}

In symbols, the semantic value of ' $\mathrm{P}$ ' would be that of a modal operator as so:

$\llbracket \mathrm{P} \rrbracket=\lambda \mathrm{p}_{<s, t>} . \lambda \mathrm{s}$. at some time before $\mathrm{s}, \mathrm{p}(\mathrm{s})=1$

Using ' $\mathrm{s}$ ' as a variable standing for times, this says that ' $\mathrm{P}$ ' denotes something which takes a function from times to true values (a temporal proposition) and returns another temporal proposition which maps a time to true provided the input proposition maps some past time to true. On this view, the object of semantic interpretation would be, essentially, a formula of Priorean tense logic.

There's another way to go, though. We are familiar in formal semantics and syntax with the idea of unprounced elements in a sentence. Thus, for example, to account for natural language quantification in a neat, compositional way, we assume that a sentence like

(5) Everybody swims

has a syntactic logical form like

\section{$\left[{ }_{s}\left[{ }_{n p}\right.\right.$ Everybody] $\left[{ }_{v p} \lambda_{1}\right.$. [ $\mathrm{t}_{1}$ swims $\left.\left.]\right]\right]$}

That is, we assume that there is a covert element, a trace (in this case ' $t_{1}{ }^{\prime}$ ), in the logical form of the sentence, which gets bound by the lambda term, so that the VP denotes a function from objects to truth values (a property), and can then serve as the input to the semantic value of 'everybody', which denotes a function from properties to truth values.

But once one has made that move of allowing unpronounced elements into the logical form to account for quantifier phrases, the option of doing the same for tense arises. After all, there's a sense in which just as 'everybody' quantifies over people, so the past tense morphology '-ed' in 'jumped' quantifies (existentially) over past times. If we had covert time denoting variables, just as we have covert object denoting variables, then we could account for the difference by positing a logical form as so: 
$\exists \mathrm{t}_{1}<\mathrm{u} . \lambda_{1}$. Carrie jump $\left(\mathrm{t}_{1}\right)$

Here ' $u$ ' stands for the utterance time, and - with a slight abuse of notation$' \exists t_{1}<u^{\prime}$ here a function from properties of times to truth values (just as 'everybody' denotes a function from properties of individuals to truth values).

One way to get at the distinction is to note that there is a question, at the level of logical form which serves as the object of semantic interpretation, of whether the quantification over times is overt or covert. According to the quantifier approach, it's overt, in the sense that there are temporal variables in the syntax which get bound. According to the operator approach, it's covert-there are no such variables, but the truth conditions of the sentence involve quantification.

One might, for all that has been said so far, think that this is a distinction without a difference. If all it comes down to is the positing or not of an anyway unpronounced piece of syntax, one might think that we don't have a very deep or important issue on our hands.

But there's more to it than that. Operators and quantifiers behave differently. Notably, a quantifier can semantically affect a variable even if another quantifier stands in its way, whereas modal operators, as standardly construed, affect the formula they immediately scope over (Schlenker 2006: 507). Compare these two formulas:

$$
\begin{aligned}
& \forall \mathrm{x} \exists \mathrm{y} \cdot \mathrm{F}(\mathrm{x}) \\
& \square \diamond \mathrm{p}
\end{aligned}
$$

The former is true provided everything in the domain of quantification is $\mathrm{F}$; the presence of the vacuous existential quantifier makes no difference to the interpretation of the formula. However, the same doesn't hold for the second formula: we can't treat the diamond as if it were vacuous, in which case the sentence would be true provided $\mathrm{p}$ was true at every world. That's just not an available reading, and it's not available because a formula is always affected by the modal which first scopes over it. Accordingly, here is a difference between modal operators, as standardly conceived, and quantifiers.

We can then ask, how do temporal expressions fit in here? Do they exhibit modal-like or quantifier-like behaviour? The linguistic data suggests the latter. There are a range of constructions involving tense well captured in terms of quantifiers but not well captured in terms of operators. Most relevant given the above is the existence of sentences in which a tense doesn't affect the intepretation of an expression in its scope. The classical example in the literature comes from Cresswell (1991):

(6) Some day, each of my students will be on the board of Linguistics And Philosophy 
Standard assumptions about scope would lead us to think that there are two disambiguations of this sentence-a temporal de dicto one, in which the tense expression 'will' scopes over the quantifier, and a temporal de re one, on which the quantifier scopes over the 'will'. Using ' $\mathrm{F}$ ' for the operator which has future existential truth conditions, we get

F (each of my students be on the board of Linguistics And Philosophy)

Each of my students F ( $\mathrm{x}$ be on the board of Linguistics and Philosophy)

The problem is that neither of these disambiguations capture the most salient reading of the sentence. The most salient reading is that at some one point in the future, each of the speaker's current students will be on the board of the journal-the speaker is imagining a situation in which his school rules supreme by capturing the editorial board. Neither of the two readings above give us this, however. The first reading gets us that at some time in the future, each of the speaker's then students will be on the board. That is, it talks about the wrong students-the future ones, which are not necessarily the current ones. The second reading has it that each of the speaker's students are such that in the future they'll be on the board-but it doesn't mean that all the students will be on the board together: one could be on the board at $t_{2}$ and only $t_{2}$, one at $t_{3}$ and only $t_{3}$, and so on. This doesn't secure the editorial board domination reading we're looking for.

On the other hand, if one were to hold that tense provides quantifiers binding temporal variables, then the situation is straightforward. We would have

$\exists \mathrm{t} 1>\mathrm{u}$. $\lambda_{1}$. Each of my students(x) be on the board $\left(\mathrm{t}_{1}\right)$ of Linguistics and Philosophy

If $x$ is a temporal variable occurring free, then it can get its value from context, so that the sentence is true provided at some future time, each of my current students is on the board of Linguistics and Philosophy then. This is the correct reading, and suggests that that the tenses behave like quantifiers rather than operators (further reading here, in addition to the paper about to be mentioned, is Schlenker 2006; Schaffer 2012; Kusumoto 2005; and references therein).

It only suggests it, however. Already in Cresswell (1991) it was realised that with sufficient complexification, one can get something like an operator view which will account for all the facts. Such a view requires the positing of a range of new and suspiciously quantifier-looking temporal operators. In the face of this, King has been lead to say,

If the complex temporal facts present in natural language are most readily and easily represented by viewing tenses as involving explicit quantification over time and as expressing relations between times, that is a good reason for thinking that tenses really work this way. 2003 . 
I agree with this. For that reason, I will mostly concentrate on quantificational analyses of tense. However, both in the interests of exhaustiveness and because at least Sider, at least sometimes, seems to be assuming an operator analysis (1996 450), I will discuss the operator theory as well. And I'll consider one other view, which is not to be found in the linguistics literature on tense but which has been suggested to me by several people, including a referee for this journal, and which indeed one can also find at Sider (1996: 450). ${ }^{1}$

\section{Against the Quantificational Analysis}

Initially, things look good for the stage theorist. I'll begin by showing how a simple modification of the quantificational theory of tense can capture a sentence like 1 from a stage-theoretic perspective.

We've already seen what the basic quantificational analysis of tense looks like. Our sentence will have the logical form like so:

\section{$\exists \mathrm{t}_{1}<\mathrm{u} . \lambda_{1}$. John be happy $\left(\mathrm{t}_{1}\right)$}

Now let's see how to modify this in the spirit of the stage theorist. The key thought behind stage theory is that a name like 'John' denotes different stages at different times. On the other hand, the stage theorist doesn't say that a predicate like 'happy' has different extensions over time: they don't need to, because it can just stand for all the happy stages, past, present and future. So an obvious thought is to say that it's names which, contrary to appearances, have a place for a variable. On this understanding, they would denote not objects, but nonconstant functions from times to stages. We'll have

\section{$\exists \mathrm{t}_{1}<\mathrm{u} . \lambda_{1}$. John $\left(\mathrm{t}_{1}\right)$ be happy}

Our sentence will be true provided there's some time prior to the time of utterance, such that applying it to the function denoted by 'John' gives a stage which belongs in the set denoted by 'happy'. And this captures the correct truth conditions: the function applied to a past time will yield a past stage of John as is required, and the sentence will be true if that stage possesses the property of happiness.

The stage theorist can appeal to a simple and well motivated modification of the semantics of tense to get the right results for basic tensed sentences. Next we'll see, however, that this modification can't account for even slightly more difficult sentences.

1. I thank a referee for pointing out that Sider seems to partially endorse this view. 


\subsection{A Problem}

Consider the following sentence:

(7) Kant was born and died in Königsberg.

The first observation to make about this sentence is that the two verbs, roughly, talk about different times: we're not asserting that there's some time such that Kant was born and died then. Rather, what we're saying is that there's some time in the past when we was born, and some other time in the past when he died.

In stage theory talk, that is to say that there's some past stage of Kant which was born, and some different past stage of Kant which died. So the sentence is really talking about two past stages. My claim is that the stage theorist can't account for this, and for a very simple reason: there's only one occurrence of 'Kant' in the sentence, so the sentence can only talk about one stage. Slightly more technically, because there's only one occurrence of 'Kant', there's only one unbound temporal variable in the sentence, which can only be bound by one of the two past tense quantifiers. But that means the one which doesn't do any binding will be semantically inert, and so the sentence will not be able to talk, as it needs to, about two different Kant stages.

And this reflects a fundamental way in which the truth conditions demanded by the metaphysics of stage theory don't fit with the semantics of natural language. For the stage theorist, the semantic point of tensed elements is to allow us to talk about past and future stages, and not, as common sense might first suggest, past and future events or states. But this is just not reflected in the syntax, in which tensed elements form syntactic constituents with adjectives and verbs, as in the adjectival phrase 'was happy' or the verb phrase 'lived', and do not form syntactic constituents with names (there's no such word as 'Kanted'). A consequence of this is that every finite verb must co-occur with some tense expression, and so when we conjoin two finite verbs, we must have two tensed elements. And this is where the problem arises, because there may be only one name for it to semantically modify.

Let me first note that there's no problem for the (unmodified) theory of tense I've given. We'll have the syntactic form, omitting brackets and labels to aid parsing

$$
\exists \mathrm{t}_{1}<\mathrm{u} \lambda_{1} . \exists \mathrm{t}_{2}<\mathrm{u} . \lambda_{2} \text {. Kant be born }\left(\mathrm{t}_{1}\right) \text { and } \operatorname{die}\left(\mathrm{t}_{2}\right)
$$

But now consider the stage theorist. The logical form must be something like

$$
\exists \mathrm{t}_{1}<\mathrm{u} \lambda_{1} . \exists \mathrm{t}_{2}<\mathrm{u} . \lambda_{2} \text {. Kant(?) be born and die }
$$

The question mark must be replaced with a trace (either $t_{1}$ or $t_{2}$ ), but it's clear that whatever quantifier we index it to, we'll get the wrong result. Neither of the two following truth conditions are correct: 
$\exists t_{1}, t_{2}<\mathrm{u}$. Kant $\left(\mathrm{t}_{1}\right)$ be born and die

$\exists \mathrm{t}_{1}, \mathrm{t}_{2}<\mathrm{u}$. Kant $\left(\mathrm{t}_{2}\right)$ be born and die

They both are true just in case the same stage of Kant possesses both the property of being born in Königsberg and of dying in Königsberg. This is not the result we were looking for.

\subsection{An Attempted Solution}

The problem arises because we don't have enough names to go around: we need two, which can be saturated by two different temporal variables, which can then each be bound by a quantifier. A natural thought is that we could resolve this issue by positing another referring expression in the syntax. The most natural thought is that it be a variable-like element which could semantically depend on the name, and in particular which could inherit the function the name denotes. That is, we could posit a syntactic form like so:

$\exists \mathrm{t}_{1}<\mathrm{u} \lambda_{1} . \exists \mathrm{t}_{2}<\mathrm{u} . \lambda_{2}$. Kant $\left(\mathrm{t}_{1}\right)$ be born and $\mathrm{o}\left(\mathrm{t}_{2}\right)$ die in Königsberg

where the variable 'o' anaphorically refers back to the function denoted by 'Kant'. This would yield truth conditions equivalent to

$$
\exists \mathrm{t}_{1}, \mathrm{t}_{2}<\mathrm{u} \text {. Born }\left(\operatorname{Kant}\left(\mathrm{t}_{1}\right)\right) \text { and } \operatorname{Died}\left(\operatorname{Kant}\left(\mathrm{t}_{2}\right)\right)
$$

Which would secure the correct result. But there are solid syntactic and semantic reasons to doubt that this approach can work. From the point of view of syntax, it's a familiar point that one can conjoin expressions of any syntactic category, as the following list indicates for names, adjectives, auxiliary verbs, and adjectives:

(8) Kant and Hegel were philosophers

(9) The table was heavy and blue

(10) You can and must do it

(11) He danced skillfully and gracefully

There is thus good reason to take the surface form of 7 seriously and hold that it is indeed a conjunction of tensed verbs. It would be surprising if one could not conjoin them, as the stage theorist seems to demand. In the same vein, it seems that we must hold this to be a case of verb conjunction to make sense of cases like

(12) Kant caught and ate a deer. 
The reason there is pressure to hold that we have a conjunctive verb phrase rather than a sentential conjunction is that both the verbs 'caught' and 'ate' are transitive and thus look for an object. But the object of 'caught', on this view, would be in a completely different sentence, and so the sentence should be as anomalous as

(13) \#Kant caught. He ate a deer.

But it isn't. Before going on, let me ward off an objection. The above sentence is bad because the syntactic object of 'caught' is not close enough for its denotation to semantically interact with the denotation of 'caught'. But it's familiar to syntacticians that there are constructions like this which are nevertheless acceptable: this is the phenomenon of right node raising introduced into the literature by Postal (1974), and exemplified by:

(14) John hates, but Mary loves, model theoretic semantics.

We here have an intransitive verb the object of which, 'model theoretic semantics', is in the wrong place, but the sentence is okay. One might think that the fact that such constructions are okay gives the stage theorist an out: they could say that what's going on here is something like right node raising. But this isn't hugely convincing: most notably, it doesn't seem like a case of right node raising. Our example 12 doesn't have the commas or intonation pattern distinctive of such constructions.

But even if that were an acceptable response to this syntactic issue, there still remains a more serious, semantic problem with the stage theorist's position: the strategy invoked here will get quantificational cases wrong. To see this, consider

(15) Everybody arrived and left safely.

In fact, to make things simpler, let's consider a tenseless example (or rather pretend that this is a tenseless example):

(16) Everybody grows and shrinks.

On the view we're considering, this is equivalent to the following, where the subscripts indicate binding:

(17) Everybody ${ }_{1}$ grows and $t_{1}$ shrinks.

But there's a problem here. In order to get the reading which we want, it must be the case that the quantifier 'everybody' binds the trace element $t_{1}$. But-at least according to orthodoxy- a universal quantifier in one sentence cannot bind a variable in another. The following is no good: 
(18)

\#Every girl arrived. Then she left.

If the covert trace strategy were correct, then we would also predict 15 to be no good. Since we don't, that's a reason to doubt the covert trace strategy, and thus the viability of the stage theorist's response to our problem.

Here's a response to the worry I just raised. There are some purported examples of cross-sentential binding of a pronoun by a universal quantifier. Here's one, from Barbara Partee via Roberts (1987):

(19) Every player choses a pawn. He puts it on square one.

One might try to argue that since such sentences are okay, we shouldn't overly worry about the fact that we're forced to posit cross-sentential binding. My response to this is to say that I doubt such sentences are okay-certainly they're much more marked and infrequent than more standard cases of binding. And I think that's sufficient to make it doubtful that the stage theorist can appeal to such cases to motivate her view: if cross sentential binding of pronouns by universal quantifiers is infrequent and marked, then sentences like 15 should be infrequent and marked, but they are neither: they are both common and very natural.

Accordingly, it appears that the semantic claims of stage theory cannot be made to cohere with the quantificational view of tense. To the extent that we follow linguists and philosophers of language who back that theory, we should reject the semantic claims of stage theory. In the next sections, I will strengthen the argument for this conclusion by showing that the semantic claims of stage theory can't be made to cohere with other views of tense.

\section{Against the Modal Operator Analysis}

The first stages in developing a modal operator analysis are clear. We make a similar move to the one we made above, only now couched in terms of intensions. Thus a standard analysis, as we've seen, would have it that names denote constant functions from times to objects, and predicates denote non-constant functions from times to extensions. The stage theorist's analysis, on the other hand, would modify this so that names denote non-constant functions from times to stages, and predicates constant functions from times to the set of all the stages, past, present or future, which satisfy the predicate.

But I think trying to develop the view any further, as in the quantificational case, will cause problems. Indeed, I think the view will be subject to a problem very similiar to the one faced by the quantificational theory. Firstly, think about what the logical form must be like. In particular, consider the contribution which 'was born and died' makes to the logical form. There are two options-either it 
contributes one tense operator, or it contributes two.

If it contributes one, we have something like

$\mathrm{P}$ (Kant be born and die in Königsberg)

This clearly gets the wrong result, as requiring that there be some one time at which Kant both was born and died. On the other hand, if it contributes two, things are not any better:

$\mathrm{P}(\mathrm{P}($ Kant be born and die in Königsberg)

This isn't the right truth conditions either: this is true provided there's a past time such that there's a time before it when Kant lived and died. It still requires that there be some one time at which Kant both lived and died, which is not what we want.

Can we get past this problem? It seems what we would need is that the two temporal operators each target one and only one verb. But since temporal operators operate on the closest expression in their scope, we'd need to have one of the operators be in the conjunction following 'and'. That is, as a first pass

\section{$\mathrm{P}($ Kant be born and $\mathrm{P}($ die in Königsberg $))$}

But that's no good-syntactically, it doesn't make sense because ' $\mathrm{P}$ ' is a sentential operator, but what follows it is not a sentence but a verb phrase. What would we need is to make what follows ' $\mathrm{P}$ ' be a sentence, and indeed a sentence which is at least truth conditionally equivalent to

Kant be born and P(Kant die in Königsberg)

This will work, at least in allowing the possibility of speaking of two different Kant stages, but it faces exactly the same problem we've gone into in detail above: there is no good reason to think that that is the structure of the sentence given to us by syntax. In particular, the conjunction that occurs in 'Kant lived and died in Königsberg' is a verb phrase conjunction and not, as required, a sentential conjunction. Ultimately, then, I think the modal operator analysis is no better than the quantificational one.

\section{Against the Predicate Analysis}

If the semantic claims of stage theory were true, then given what we know about the semantics of tense, we should expect that they could be spelled out in terms either of the operator or the quantifier theory of tense. Given that this is not so, we should lower our credence in the semantic claims of stage theory. 
However, it remains possible that such claims could be made true in a different way, with a different view about the semantics of tense. In this section I want to consider a view which several people, including reviewers for this journal, have suggested has potential. Indeed, it can arguably found in Sider's (1996: 450) comments on de re temporal predication. He considers a sentence like

(20) Clinton was once governer of Arkansas.

and suggests that we should analyse it as a predicating, of Clinton, the property of previously being governer of Arkansas. That is, the tense in 'was' functions to form a complex predicate, and does not, as on the other analyses, contribute either a quantifier or an operator, with a semantic value that looks as so:

«was governer of Akarkansas $\rrbracket=\lambda \mathrm{s}$. there's a past stage I-related to $\mathrm{s}$ which possesses the being the governer of Arkansas

The name 'Clinton', in turn, when uttered at any given time, picks out the stage of Clinton at that time.

On this view, it's not that there are tenseless verbs which get acted upon by either being associated with bound temporal variables (on the quantifier view of tense) or by being in the scope of temporal operators (on the operator view). Rather, I think the best way to understand this view is that tense carrying expressions like 'was' or the past tense morpheme '-ed' are predicate modifiers, that is, expressions which take a predicate to yield a predicate.

This view, it should be clear, will resolve the problem which this paper is centred around. The truth conditions of 7 will be that it's true provided Kant has a past stage which was born in Königsberg, and a past stage which died in Königsberg, and this is exactly the right result.

So far, so good. But I think a little attention will show us why this is ultimately not a viable account of how temporal information gets expressed in natural language. The crucial observation that will enable us to see this is that the tense of a verb systematically affects other elements of the sentence, but predicate modifiers don't behave like this.

An example will show this. I'm a warden talking about a prison break that happened this morning. Consider two scenarios: in the first, the prisoners who tried to escape were caught. I describe this situation by saying,

(21) Every fugitive was recaptured.

In the second scenario, I have been less successful: I didn't recapture any prisoners. I utter,

(22) Every fugitive was born in Toronto. 
These are both perfectly fine, natural sentences. But they're notably different. In the first, we are talking about former fugitives who have now been caught. That is to say, we are using an expression 'fugitives' to pick out not the current fugitives, but past fugitives. By contrast, in the second scenario, we are talking about current fugitives. Any theory of tense must respect the fact that we can get both readings.

Now the reader's first thought would be that we can explain this in terms of scope. In our first sentence, the tense introduced by 'was' scopes over the universal quantifier, and in the second, the quantifier scopes over the tense.

But I just don't see how to make sense of that on this view without collapsing it into either an operator or a quantificational theory. The key reason why the quantificational and operator theory can appeal to scope is because on such views tenses, although apparently predicative, actually introduce expressions which apply to sentences, namely either quantifiers with times in their domain or operators which operate on temporal propositions. If the predicate view is to be different-and it needs to be, because the other two views don't work-it can't say anything like this.

But then it won't be the case that we can account for both sentences. In particular, 21 will be the problem. The truth conditions we can get for it is that every fugitive is such that they have a past temporal part which possesses the property of being recaptured. But this is-absent any further story-talking about the current fugitives. That's not what we want-we want to talk about the people who were fugitives at the time the verb is, intuitively speaking, 'about', namely the time of the recapturing. That is to say, we want the interpretation of the verb to affect that of the noun phrase 'every fugitive'. I just don't see how this can be if 'was recaptured' simply expresses the property of having previously been captured. ${ }^{2}$ Accordingly, I don't think the predicate analysis is viable.

Before finishing, let me say something about David Lewis's counterpart theory of modality. A referee suggested the following worry with my paper: if my argument is right, then it should apply also to counterpart theory. In particular, if we were to try to develop a formal semantics for natural language in the spirit of counterpart theory, a sentence like 'Kant could have been a musician and could have been a singer' would run into exactly the same problem I presented above: we would need to look to two counterparts, but there is only one occurrence of the name 'Kant' in the sentence. Given this, it might seem strange that I didn't urge this as an objection to counterpart theoretic semantics more generally, be they temporal or alethic.

There are several reasons why I wrote the paper I did. The main one is that

2. And note that in the Sider passage just mentioned, to deal with an essentially similar issue, he explicitly introduces sentential past tense operators; we've seen above that that move won't work. 
I felt less sure about the data in the case of alethic modality: while it's beyond doubt that 'Kant lived and died in Königsberg' is a fine sentence, 'Kant could have been a musician and could have been a singer' is a bit clunky. Second, the syntax is also cleaner in the temporal case: it's again beyond doubt that tensed elements like '-ed' operate directly on verbs, and while I think it's also clear that modal auxilaries like 'could' or 'might' do so too, it's more open to doubt (we might be tempted to say that there's a covert big PRO somewhere, for example). For these reasons I concentrated on stage theory. Nevertheless, I am tempted to think that the line of argument developed here does apply also to modal counterpart theory; but that's work for another paper.

\section{Conclusion}

The stage theorist, then, can't deal with our simple sentence. One might think that I haven't conclusively shown this-that it's possible that with sufficient ingenuity, the friend of stages will be able to come up a theory of tense that can avoid the problem I've posed. But I think there's good reason to be pessimistic about this. For stage theory's inability to deal with our sentence derives from a deep and fundamental fact about language, namely, and as we've seen, that tense elements modify verbs and not noun phrases. But the stage theorist is compelled to say that such elements semantically work on noun phrases, to enable us to talk about past and future stages of objects. This mismatch between what stage theorists require of the semantics of tense, and what natural language actually delivers, renders stage theory vulnerable to problems like the one I've suggested, and supplies a good reason to disbelieve it.

\section{Acknowledgements}

I would like to thank Herman Cappelen, Josh Dever, Derek Ball, Ofra Magidor, audiences at the 2015 SPA in Glasgow, the 2016 Joint session in Cardiff, Arché, and CSMN, and several anonymous referees and the Ergo area editor for helpful comments on the material in this paper. I would also like the thank the AHRC and the Royal Institute Of Philosophy for funding this research.

\section{References}

Cresswell, M. J. (1991). Entities and Indicies. Kluwer.

Goranko, Valentin and Antony Galton (2015). Temporal Logic. In Edward N. Zalta (Ed.), The Stanford Encyclopedia of Philosophy (Winter 2015 ed.). Retrieved from https://plato.stanford.edu/archives/win2015/entries/ 


\section{logic-temporal/}

Hawley, Katherine (2001). How Things Persist. Oxford University Press.

King, Jeffrey C. (2003). Tense, Modality, and Semantic Values. Philosophical Perspectives, $17(1), 195^{-246 .}$

Kusumoto, Kiyomi (2005). On the Quantification Over Times in Natural Language. Natural Language Semantics, 13(4), 317-357.

Postal, Paul Martin (1974). On Raising: One Rule of English Grammar and Its Theoretical Implications. MIT Press.

Prior, A. N. (1968). Papers on Time and Tense. Oxford University Press.

Roberts, Craige (1987). Modal Subordination, Anaphora, and Distributivity (Unpublished doctoral dissertation). University of Massichusetts Amherst.

Schaffer, Jonathan (2012). Necessitarian Propositions. Synthese, 189(1), 119-162.

Schlenker, Philippe (2006). Ontological Symmetry in Language: A Brief Manifesto. Mind and Language, 21(4), 504-539.

Sider, Theodore (1996). All the World's a Stage. Australasian Journal of Philosophy, 74(3), 433-453.

Sider, Theodore (2001). Four Dimensionalism: An Ontology of Persistence and Time. Oxford University Press. 